\title{
Investigation of layered and porous nanomaterials by electron diffraction tomography
}

Yasar Krysiak $^{1}$, Haishuang Zhao ${ }^{1}$, Bastian Barton ${ }^{1}$, Jürgen Senker ${ }^{2}$, Reinhard B. Neder ${ }^{3}$, Ute Kolb ${ }^{1}$ ${ }^{1}$ Inst. Of Inorg. Chemistry And Analyt. Chemistry, Johannes Gutenberg University, Mainz, Germany, ${ }^{2}$ Inorganic Chemistry III, University of Bayreuth, Bayreuth, Germany, ${ }^{3}$ Chair for Crystallography and Structural Physics, Friedrich-Alexander-Universität Erlangen-Nürnberg, Erlangen, Germany E-mail: krysiak@uni-mainz.de

Crystal structure solution using automated electron diffraction tomography [1] (ADT) is nowadays an accepted method for deriving full 3D structure information at atomic resolution from a nanocrystalline material. Crystals with special structural features like twinning, super-structural effects or pseudo-symmetry could be solved ab-initio with ADT. In case of a reduced periodicity in the lattice due to electron radiation damage, disorder or stress/strain effects the intensities are more and more difficult or impossible to derive from diffraction patterns. Uncertainties in the intensity extraction allow often the detection of the major structure but cause problems for detailed structural insights being especially important for materials physical properties.

Currently, we aim for a more detailed description of nanomaterial structures, including non-periodic effects through a combination of different methods like TEM imaging, ADT and structure simulation and modelling. ADT data can be directly used for an ab-initio structure solution of the average structure. Disorder and distortion or intergrown phases are simulated using the DISCUS software package [2] through accurate analysis of the structure's variance. The results are compared to TEM holograms [3] reconstructed from focal series.

In such a way, the intergrown polymorphs $A$ and $B$ of Zeolite beta could be solved ab-initio from one single ADT dataset by integrating the relative reflection intensities with two distinct lattices. The disorder type was identified by exit wave reconstruction and confirmed by DISCUS simulations. The application of this approach was used to solve the non-periodic structure of RUB-5, revealing an unknown zeolite framework, as well as of its precursor RUB-6.

A further successful method combination used to determine the $\mathrm{H} / \mathrm{Li}$ substructure of poly (triazinimide) / LiCl was to combine nuclear magnetic resonance (NMR) measurements and the analysis of X-ray total scattering (PDF) with ADT results. This sample with incorporated lithium chloride recently attracted substantial attention due to its photocatalytic activity for water splitting. In addition to the known major structure, electron diffraction tomography revealed the positions of the Li atoms and a symmetry reduction. NMR simulations added information about the protonation of the channels and the PDF analysis of X-ray powder diffraction data, simulated with DISCUS, proposed a long-range modulation of the layers.

The 3D atomic structure of different compounds forming highly distorted and disordered nanoparticles could be successfully described by combining ADT, layer shift simulations, TEM imaging and further techniques.

[1] Mugnaioli, E., Gorelik, T. \& Kolb, U. (2009). Ultramicroscopy. 109, 758-765.

[2] Proffen, T. \& Neder, R. B. (1997). J. Appl. Crystallogr. 30, 171-175.

[3] Barton, B., Jiang, B. \& Kisielowski, C. (2012). Microsc. Microanal. 18, 982-994.

Keywords: disorder simulation, electro diffraction tomography, in-line electron holography 